\title{
Differential Modulation for Bi-directional Relaying with Analog Network Coding
}

\author{
Lingyang Song, Yonghui Li, Anpeng Huang, Bingli Jiao, \\ and Athanasios V. Vasilakos
}

\begin{abstract}
In this paper, we propose an analog network coding scheme with differential modulation (ANCDM) using amplify-and-forward protocol for bidirectional relay networks when neither the source nodes nor the relay knows the channel state information (CSI). The performance of the proposed ANC-DM scheme is analyzed and a simple asymptotic bit error rate (BER) expression is derived. The analytical results are verified through simulations. It is shown that the BER performance of the proposed differential scheme is about $3 \mathrm{~dB}$ away from that of the coherent detection scheme. To improve the system performance, the optimum power allocation between the sources and the relay is determined based on the simplified BER. Simulation results indicate that the proposed differential scheme with optimum power allocation yields 1-2 dB performance improvement over an equal power allocation scheme.
\end{abstract}

\section{Index Terms}

Differential modulation, bi-directional relaying, analog network coding, amplify-and-forward protocol

\section{INTRODUCTION}

Bi-directional relay communication has attracted considerable interest recently [1]-[6], and various bi-directional relay protocols for wireless systems have been proposed [1]-[3].

Copyright (c) 2010 IEEE. Personal use of this material is permitted. However, permission to use this material for any other purposes must be obtained from the IEEE by sending a request to pubs-permissions@iee.org.

This work was partially supported by the National Natural Science Foundation of China under Grant number 60972009 and 60811130529.

Lingyang Song, Bingli Jiao and Anpeng Huang are with Peking University, China (e-mail: lingyang.song@pku.edu.cn,jiaobl@pku.edu.cn,hapku@pku.edu.cn).

Yonghui Li is with University of Sydney, Australia (e-mail: lyh@ieee.org).

Athanasios V. Vasilakosis is with University of Western Macedonia, Greece (e-mail: vasilako@ath.forthnet.gr). 
In [1] [2], the conventional network coding scheme is applied to the bi-directional relay network. Two source nodes transmit to the relay, separately. The relay decodes the received signals, performs binary network coding, and then broadcasts network coded symbols back to both source nodes. However, this scheme may cause irreducible error floor due to the detection errors which occur at the relay node.

In [3]-[5], an amplify and forward based network coding scheme, referred to as the analog network coding, was proposed. In this scheme, both source nodes transmit at the same time so that the relay receives a superimposed signal. The relay then amplifies the received signal and broadcasts it to both source nodes. Analog network coding is particularly useful in wireless networks as the wireless channel acts as a natural implementation of network coding by summing the wireless signals over the air.

Almost all existing works in bi-directional relay communications using analog network coding assume that the sources and the destination have perfect knowledge of channel state information (CSI) for all transmission links. As a result, coherent detection can be readily employed at either sources or relay, or both [1]-[5]. In some scenarios, e.g. the slow fading environment, the CSI is likely to be acquired by the use of pilot symbols. However, when the channel coefficients vary fast, channel estimation may become difficult. In addition, the channel estimation increases computational complexity in the relay node and reduces the data rate. Moreover, it would be difficult for the destination to acquire the source-torelay channel perfectly through pilot signal forwarding without noise amplification. Hence, differential modulation without need of any CSI would be a practical solution.

In a differential bi-directional relay network, each source receives a superposition of differentially encoded signals from the other source, and it has no knowledge of CSI of both channels. All these problems present a great challenge for designing differential modulation schemes in two-way relay channels. In [6], differential receivers for differential two-way relaying were presented using analog network coding. These non-coherent schemes were realized by averaging the channel coefficients of the substraction of adjacent received signals. However, the approaches may result in more than $3 \mathrm{~dB}$ performance loss compared to the coherent schemes due to instantaneous detection errors.

In this paper, we propose an analog network coding scheme with differential modulation (ANC-DM) using amplify-and-forward protocol for bidirectional relay networks so that the CSI is not required at both sources and the relay. The performance of the proposed ANC-DM scheme is analyzed and a simple asymptotic bit error rate (BER) expression is 
derived. The analytical results are verified through simulations. They show that the proposed differential scheme is about $3 \mathrm{~dB}$ away compared to the coherent detection scheme. To improve the system performance, the optimum power allocation between the sources and the relay is determined based on the provided simplified BER. Simulation results show that optimum power allocation yields 1-2 $\mathrm{dB}$ performance improvement over an equal power allocation scheme.

Note that unlike [6], the destination realizes differential detection by subtracting away its own contribution in the received signals based on the power estimation. Besides, our scheme requires linear complexity, while the detectors in [6] are much complex and non-linear which may calculate the modified Bessel function of second kind. Note also that the application of differential modulation for the bi-directional relaying in [1] [2] with digital network coding is relatively straightforward as the received signals at the relay from the sources can be decoded separately due to the use of orthogonal transmissions. However, for amplify-andforward relaying [3]-[5], the relay receives a superposition of differentially encoded signals from the sources which makes the final detection at the source difficult.

The rest of the paper is organized as follows: In Section II, we describe the proposed differential modulation scheme. Theoretical analysis is given in Section III. Section IV presents the optimal transmit power allocation between the sources and the relay. Simulation results are provided in Section V. In Section VI, we draw the main conclusions.

Notation: Boldface lower-case letters denote vectors, $(\cdot)^{*}$ stands for complex conjugate, $(\cdot)^{T}$ represents transpose, $\mathbb{E}$ is used for expectation, Var represents variance, $\|\mathbf{x}\|^{2}=\mathbf{x}^{H} \mathbf{x}$, and $\mathfrak{R}(\cdot)$ denotes real part.

\section{DifFERENTIAL MODUlation FOR BIDIRECTIONAL RELAY NETWORKS}

\section{A. Differential Encoding}

We consider a three-node bi-directional relay network consisting of two source nodes, denoted by $S_{1}$ and $S_{2}$, and one relay node, denoted by $R$. All nodes are equipped with one antenna and operate in a half-duplex way so that the complete transmission can be divided into two phases, as shown in Fig. 1. In the first phase, both source nodes simultaneously send the differentially encoded information to the relay, and in the second phase, the relay broadcasts the combined signals to both sources. Let $c_{1}(t) \in \mathcal{A}$ denote the symbol to be transmitted by the source $S_{1}$ at the time $t$, where $\mathcal{A}$ represents a unity power $M$-PSK constellation set. In 
the differential modulation bi-directional relay system, the signal $s_{1}(t)$ sent by the source $S_{1}$ is given by

$$
s_{1}(t)=s_{1}(t-1) c_{1}(t), \quad c_{1}(t) \in \mathcal{A}
$$

Similarly, the signal transmitted by $S_{2}$ at the time $t$ is given by

$$
s_{2}(t)=s_{2}(t-1) c_{2}(t), \quad c_{2}(t) \in \mathcal{A}
$$

\section{B. Differential Decoding}

In the bi-directional relayed transmission, the source nodes first broadcast the information to the relay. For simplicity, we assume that the fading coefficients are constant over one frame of length $L$, and change independently from one frame to another. The received signal in the relay at time $t$ can be expressed as

$$
y_{r}(t)=\sqrt{p_{1}} h_{1} s_{1}(t)+\sqrt{p_{2}} h_{2} s_{2}(t)+n_{r}(t)
$$

where $p_{1}$ and $p_{2}$ represent the transmit power at $S_{1}$ and $S_{2}$ respectively, $h_{1}$ and $h_{2}$ are the Rayleigh fading coefficients with zero mean and unit variance between $S_{1}$ and $R$, and between $S_{2}$ and $R$, respectively, $n_{r}(t)$ denotes zero mean complex Gaussian random variable with two sided power spectral density of $N_{0} / 2$ per dimension, and we furthermore assume $S_{1}, S_{2}$, and $R$ have the same noise variance.

In the second phase, the relay $R$ amplifies $y_{r}(t)$ by a factor $\beta$ and then broadcasts its conjugate, denoted by $y_{r}^{*}(t)$, to both $S_{1}$ and $S_{2}$. The corresponding signal received by $S_{1}$ at time $t$, denoted by $y_{1}(t)$, can be written as

$$
\begin{aligned}
y_{1}(t) & =\beta \sqrt{p_{r}} h_{1} y_{r}^{*}(t)+n_{1}(t) \\
& =\mu s_{1}^{*}(t)+\nu s_{2}^{*}(t)+w_{1}(t),
\end{aligned}
$$

where $\beta=\left(p_{1}\left|h_{1}\right|^{2}+p_{2}\left|h_{2}\right|^{2}+N_{0}\right)^{-\frac{1}{2}}, p_{r}$ represents the transmit power by the relay, $\mu \triangleq$ $\beta \sqrt{p_{1} p_{r}}\left|h_{1}\right|^{2}, \nu \triangleq \beta \sqrt{p_{2} p_{r}} h_{1} h_{2}^{*}$, and $w_{1}(t) \triangleq \beta \sqrt{p_{r}} h_{1} n_{r}^{*}(t)+n_{1}(t)$. Note that unlike traditional ANC schemes [3]-[5], $y_{r}^{*}(t)$ is transmitted from the relay, which obviously yields $\mu>0$. The reason of doing this is to make the receiver easily estimate $\mu$ and then subtract $\mu s_{1}^{*}(t)$ in (4) for differential detection.

As the relay has no CSI, the normalization factor $\beta$ has to be obtained indirectly. We may rewrite the received signals in (3) in a vector format, given by

$$
\mathbf{y}_{r}=\sqrt{p_{1}} h_{1} \mathbf{s}_{1}+\sqrt{p_{2}} h_{2} \mathbf{s}_{2}+\mathbf{n}_{r}
$$


where $\mathbf{y}_{r}=\left[y_{r}(1), \ldots, y_{r}(L)\right]^{T}, \mathbf{s}_{1}=\left[s_{1}(1), \ldots, s_{1}(L)\right]^{T}, \mathbf{s}_{2}=\left[s_{2}(1), \ldots, s_{2}(L)\right]^{T}$, and $\mathbf{n}_{r}=\left[n_{r}(1), \ldots, n_{r}(L)\right]^{T}$. To estimate the average receive power, we multiply the received signals by its Hermitian transpose as

$$
\begin{aligned}
\left\|\mathbf{y}_{r}\right\|^{2}=p_{1}\left|h_{1}\right|{ }^{2} \mathbf{s}_{1}^{H} \mathbf{s}_{1} & +p_{2}\left|h_{2}\right|^{2} \mathbf{s}_{2}^{H} \mathbf{s}_{2}+2 \sqrt{p_{1} p_{2}} \mathfrak{R}\left\{h_{1}^{*} h_{2} \mathbf{s}_{1}^{H} \mathbf{s}_{2}\right\} \\
& +2 \sqrt{p_{1}} \mathfrak{R}\left\{h_{1} \mathbf{n}_{r}^{H} \mathbf{s}_{1}\right\}+2 \sqrt{p_{2}} \mathfrak{R}\left\{h_{2} \mathbf{n}_{r}^{H} \mathbf{s}_{2}\right\}+\mathbf{n}_{r}^{H} \mathbf{n}_{r}
\end{aligned}
$$

By taking the expectation of (6), $\beta$ can be then approximated at high SNR by

$$
\beta=\sqrt{\frac{\mathbb{E}\left\{\mathbf{y}_{r}^{H} \mathbf{y}_{r}\right\}}{L}} \approx \sqrt{\frac{\left\|\mathbf{y}_{r}\right\|^{2}}{L}},
$$

where $\mathbb{E}\left\{\mathbf{s}_{1}^{H} \mathbf{s}_{1}\right\}=\mathbb{E}\left\{\mathbf{s}_{2}^{H} \mathbf{s}_{2}\right\}=L, \mathbb{E}\left\{\mathbf{n}_{r}^{H} \mathbf{n}_{r}\right\}=L N_{0}$, and $\mathbb{E}\left\{\mathbf{s}_{1}^{H} \mathbf{s}_{2}\right\}=\mathbb{E}\left\{\mathbf{n}_{r}^{H} \mathbf{s}_{1}\right\}=\mathbb{E}\left\{\mathbf{n}_{r}^{H} \mathbf{s}_{2}\right\}=$ 0 .

Similarly, the received signal at $S_{2}$ can be calculated as

$$
y_{2}(t)=\beta \sqrt{p_{r}} h_{2} y_{r}^{*}(t)+n_{2}(t),
$$

As $S_{1}$ and $S_{2}$ are mathematically symmetrical, as shown in (4) and (8), for simplicity, we in the next only discuss the decoding as well as the corresponding theoretical analysis for signals received by $S_{1}$.

Recalling the differential encoding process in (2), (4) can be further written as

$$
\begin{aligned}
y_{1}(t) & =\mu s_{1}^{*}(t)+\nu s_{2}^{*}(t)+w_{1}(t), \\
& =\mu s_{1}^{*}(t)+\nu s_{2}^{*}(t-1) c_{2}^{*}(t)+w_{1}(t),
\end{aligned}
$$

Obviously, since $s_{1}(t)$ is known, to decode $c_{2}(t)$ in (9), $\mu s_{1}^{*}(t)$ should be removed. To do this, we have to first estimate $\mu$, where $\mu>0$.

Similar to (10), the received signal in (4) can be expressed in a vector form as follows

$$
\mathbf{y}_{1}=\mu \mathbf{s}_{1}+\nu \mathbf{s}_{2}+\mathbf{w}_{1},
$$

where $\mathbf{y}_{1}=\left[y_{1}(1), \ldots, y_{1}(L)\right]^{T}$ and $\mathbf{w}_{1}=\left[w_{1}(1), \ldots, w_{1}(L)\right]^{T}$. At high SNR, we may approximately obtain

$$
\mu^{2}+|\nu|^{2} \approx \mathbf{y}_{1}^{H} \mathbf{y}_{1} / L
$$

Since the source node $S_{1}$ can retrieve its own information $s_{1}(t-1)$ and $c_{1}(t)$, based on (1) and (9), we have the following transformation

$$
\begin{aligned}
\widetilde{y}_{1}(t) & \triangleq c_{1}^{*}(t) y_{1}(t-1)-y_{1}(t) \\
& =\nu s_{2}^{*}(t-1)\left(c_{1}(t)-c_{2}(t)\right)^{*}+\widetilde{w}_{1}(t),
\end{aligned}
$$


where $\widetilde{w}_{1}(t) \triangleq c_{1}^{*}(t) w_{1}(t-1)+w_{1}(t)$. Then, $|\nu|^{2}$ can be approximately calculated in a similar way as (11)

$$
|\nu|^{2} \approx \frac{\widetilde{\mathbf{y}}_{1}^{H} \widetilde{\mathbf{y}}_{1}}{L \mathbb{E}\left[\left|s_{2}(t-1)\right|^{2}\right] \mathbb{E}\left[\left|c_{1}(t)-c_{2}(t)\right|^{2}\right]},
$$

where $\widetilde{\mathbf{y}}_{1}=\left[\widetilde{y}_{1}(1), \ldots, \widetilde{y}_{1}(L-1)\right]^{T}, \mathbb{E}\left[\left|s_{2}(t-1)\right|^{2}\right]=1$, and the calculation of $\mathbb{E}\left[\mid c_{1}(t)-\right.$ $\left.\left.c_{2}(t)\right|^{2}\right]$ is given in Section-II-C. As $\mu$ is positive, by combining (7), (11) and (13), we have

$$
\mu \approx \begin{cases}\sqrt{\Delta}, & \Delta>0 \\ 0, & \Delta \leq 0\end{cases}
$$

where $\Delta \triangleq \frac{\mathbf{y}_{1}^{H} \mathbf{y}_{1}}{L}-\frac{\widetilde{\mathbf{y}}_{\mathbf{1}}^{H} \widetilde{\mathbf{y}}_{1}}{L \mathbb{E}\left[\left|s_{2}(t-1)\right|^{2}\left|c_{1}(t)-c_{2}(t)\right|^{2}\right]}$. Note that in low SNR, $\Delta$ could be negative due to the noise effect, and thus we set $\mu \approx 0$ instead. The estimation method given in (14) is evaluated in Fig. 2 ,

By subtracting $\mu s_{1}^{*}(t)$, (9) can be further written as

$$
\begin{aligned}
y_{1}^{\prime}(t) & \triangleq y_{1}(t)-\mu s_{1}(t) \\
& =\nu s_{2}^{*}(t-1) c_{2}^{*}(t)+w_{1}(t) \\
& =\left(y_{1}^{\prime}(t-1)-w_{1}(t-1)\right) c_{2}^{*}(t)+w_{1}(t),
\end{aligned}
$$

Finally, the following linear decoder can be used to recover $c_{2}(t)$

$$
\widetilde{c}_{2}(t)=\underset{c_{2}(t) \in \mathcal{A}}{\arg \max }\left\{y_{1}^{\prime}(t) y_{1}^{\prime *}(t-1) c_{2}(t)\right\} .
$$

And $c_{1}(t)$ can be differentially decoded in a similar way by the source $S_{2}$. Note that in comparison to traditional differential modulation [7], the extra complexity comes from the $\mu$ estimation in (14), which is linear and only comprises a few number of additions and multiplications. But the receiver in [6] requires complicated computations, such as the zerothorder modified Bessel function of the second kind.

By ignoring the second order term, the corresponding SNR of the proposed differential detection scheme can be written as

$$
\begin{aligned}
\gamma_{d} & \approx \frac{|\nu|^{2}}{2 \operatorname{Var}\left\{w_{1}(t)\right\}} \\
& \approx \frac{\beta^{2} p_{2} p_{r}\left|h_{1}\right|^{2}\left|h_{2}\right|^{2}}{2\left(\beta^{2} p_{r} N_{0}\left|h_{1}\right|^{2}+N_{0}\right)} \\
& \approx \frac{\psi_{2} \psi_{r}\left|h_{1}\right|^{2}\left|h_{2}\right|^{2}}{2\left(\left(\psi_{1}+\psi_{r}\right)\left|h_{1}\right|^{2}+\psi_{2}\left|h_{2}\right|^{2}+1\right)}
\end{aligned}
$$

where $\operatorname{Var}\left\{w_{1}(t)\right\}=\beta^{2} p_{r} N_{0}\left|h_{1}\right|^{2}+N_{0}, \psi_{1} \triangleq p_{1} / N_{0}, \psi_{2} \triangleq p_{2} / N_{0}$, and $\psi_{r} \triangleq p_{r} / N_{0}$. 
Alternatively, if coherent detection is used, under the assumption of $h_{1}, h_{2}$ and $N_{0}$ available at $S_{1}$, after subtracting $\mu s_{1}(t)^{*}$ from $y_{1}(t)$ in (4), the corresponding SNR can be calculated as

$$
\begin{aligned}
\gamma_{c} & \triangleq \frac{|\nu|^{2}}{\operatorname{Var}\left\{w_{1}(t)\right\}} \\
& =\frac{\psi_{2} \psi_{r}\left|h_{1}\right|^{2}\left|h_{2}\right|^{2}}{\left(\psi_{1}+\psi_{r}\right)\left|h_{1}\right|^{2}+\psi_{2}\left|h_{2}\right|^{2}+1} .
\end{aligned}
$$

By comparing (17) and (18), we can easily obtain

$$
\gamma_{d} \approx \frac{\gamma_{c}}{2}
$$

which clearly indicates that the differential detector in (16) suffers around $3 \mathrm{~dB}$ performance loss compared to the coherent scheme.

\section{The Calculation of $\mathbb{E}\left[\left|c_{1}(t)-c_{2}(t)\right|^{2}\right]$ in (13)}

From (13), the average power of $c_{1}(t)-c_{2}(t)$ needs to be calculated. When $M$-PSK constellations are applied, the number of symbols produced in the new constellation by $c_{1}(t)-c_{2}(t)$ is finite. Hence, it is easy to derive the average power of the new constellation sets. Note that the value of $c_{1}(t)-c_{2}(t)$ can be equal to zero, which may affect the estimation accuracy in (13).

In order to overcome this problem, we may properly choose a rotation angle for the symbol modulated in source $S_{2}$ by $c_{2}(t) e^{-j \theta}$, ensuring that $c_{1}(t)-c_{2}(t)$ in (13) is nonzero. For a $M$-PSK constellation, the effective rotation angle is in the interval $[-\pi / M, \pi / M]$ from the symmetry of symbols. For a regular and symmetrical constellation, the rotation angle may be simply set as $\theta=\pi / M$. Similar approach may be used to generate the rotation angle for other types of constellations.

Here, we give two examples on how to compute the average symbol power:

1. Supposing BPSK constellation $\{-1,1\}$ is used, we have $c_{1}(t)-c_{2}(t) \in\{-2,0,2\}$. Hence, the calculation of average power in the new set is straightforward.

2. Supposing $S_{1}$ uses the BPSK set $\{-1,1\}$, by constellation rotation, $S_{2}$ can use $\{-j, j\}$. And we can get $c_{1}(t)-c_{2}(t) \in\{-1-j,-1+j, 1-j, 1+j\}$. Hence, it is also easy to derive the average power in the new constellation set. 


\section{PeRformance AnAlysis}

For simplicity, we in this section analyze the BER performance using BPSK for the proposed ANC-DM scheme, and we assume $p_{1}=p_{2}=p_{s}$ and $p_{s}=\lambda p_{r}$, where $\lambda>0$, and thus, $\psi_{1}=\psi_{2}=\psi_{s}=\lambda \psi_{r}$. (17) can be rewritten as

$$
\gamma_{d} \approx \frac{\psi_{s} \psi_{r}^{\prime}\left|h_{1}\right|^{2}\left|h_{2}\right|^{2}}{2(1+\lambda)\left(\psi_{r}^{\prime}\left|h_{1}\right|^{2}+\psi_{s}\left|h_{2}\right|^{2}+1\right)}
$$

where $\psi_{r}^{\prime} \triangleq(1+\lambda) \psi_{r}$.

Let $X=\gamma_{d}$, and the BER for BPSK modulation can generally be expressed as

$$
\mathrm{BER}=\mathbb{E}[Q(2 X)]=\frac{1}{2 \sqrt{\pi}} \int_{0}^{\infty} \frac{\exp (-x) F_{X}(x)}{\sqrt{x}} \mathrm{~d} x,
$$

where $Q(\cdot)$ is the Gaussian-Q function, $F_{X}(x)$ is the cumulative distribution function (CDF) of $X$. The right side of the equation can be readily obtained by integration by parts. The above expression is useful as it allows us to obtain the BER directly in terms of the CDF of $X$.

By using a general result from [8], the BER in (21) can be approximated in the high SNR regime by considering a first order expansion of the CDF of $X$. Specifically, if the first order expansion of the CDF of $X$ can be written in the form

$$
F_{X}(x)=\frac{\alpha x^{N+1}}{\bar{\lambda}^{N+1}(N+1)}+o\left(x^{N+1+\varepsilon}\right), \varepsilon>0,
$$

where $\bar{\lambda}$ represents the average transmit SNR. At high SNR, the asymptotic BER is given by [8]

$$
\mathrm{BER}=\frac{\alpha \Gamma\left(N+\frac{3}{2}\right)}{2 \sqrt{\pi} \bar{\lambda}^{N+1}(N+1)}+o\left((\bar{\lambda})^{-(N+1)}\right) .
$$

The PDF of $X$ can be obtained with the help of [9]

$$
\begin{aligned}
P_{X}(x)=\frac{8(1+\lambda)^{2} x \exp \left(-2(1+\lambda)\left(\psi_{s}^{-1}+\psi_{r}^{\prime-1}\right) x\right)}{\psi_{s} \psi_{r}^{\prime}}\left[\frac{\psi_{s}+\psi_{r}^{\prime}}{\sqrt{\psi_{s} \psi_{r}^{\prime}}} \times K_{1}\left(\frac{4(1+\lambda) x}{\sqrt{\psi_{s} \psi_{r}^{\prime}}}\right)\right. \\
\left.+2 K_{0}\left(\frac{4(1+\lambda) x}{\sqrt{\psi_{s} \psi_{r}^{\prime}}}\right)\right] U(2(1+\lambda) x),
\end{aligned}
$$

where $K_{0}(\cdot)$ and $K_{1}(\cdot)$ are the zeroth-order and first-order modified Bessel functions of the second kind, respectively, and $U(\cdot)$ is the unit step function. Note that the exact BER, which is complicated in computation, does not have a closed-form solution. However, at high SNR, when $z$ approaches zeros, the $K_{1}(z)$ function converges to $1 / z$ [10], and the value of 
the $K_{0}(z)$ function is comparatively small, which could be ignored for asymptotic analysis. Hence, $P_{X}(x)$ in (24) can be approximated as

$$
P_{X}(x) \approx \frac{2(1+\lambda)\left(\psi_{s}+\psi_{r}^{\prime}\right) \exp \left(-2(1+\lambda)\left(\psi_{s}^{-1}+\psi_{r}^{\prime-1}\right) x\right)}{\psi_{s} \psi_{r}^{\prime}} .
$$

For the ANC-DM, the CDF of destination SNR $\gamma_{d}$ can be approximated as

$$
\begin{aligned}
F_{X}(x) & \approx 1-\exp \left(-2(1+\lambda)\left(\psi_{s}^{-1}+\psi_{r}^{\prime-1}\right) x\right) \\
& \approx 2(1+\lambda)\left(\psi_{s}^{-1}+\psi_{r}^{\prime-1}\right) x+o\left(x^{1+\varepsilon}\right) .
\end{aligned}
$$

Finally, comparing (26) with (22) and (23), the asymptotic BER of ANC-DM at high SNR can be approximated as

$$
\begin{aligned}
\mathrm{BER} & \approx \frac{2(1+\lambda) \Gamma\left(\frac{3}{2}\right)}{2 \sqrt{\pi}}\left(\psi_{s}^{-1}+\psi_{r}^{\prime-1}\right) \\
& =\frac{(1+\lambda)\left(\psi_{s}^{-1}+\psi_{r}^{\prime-1}\right)}{2} .
\end{aligned}
$$

\section{Transmit Power Allocation}

In this section, we discuss how to allocate power to both sources and the relay subject to total transmission power constraint. It can be seen from (27) that the asymptotic BER of the proposed differential modulation scheme depends non-linearly upon $p_{s}$ and $p_{r}$. Hence, when the total transmit power is fixed, $2 p_{s}+p_{r}=p$, the power allocation problem over Rayleigh channels can be formulated to minimize the asymptotic BER at high SNR in (27)

$$
\min \text { BER }
$$

$$
\text { s.t. } 2 p_{s}+p_{r}=p \quad\left(0<p_{s}<p, 0<p_{r}<p\right)
$$

where we assume $p_{1}=p_{2}=p_{s}$ and $p_{s}=\lambda p_{r}$.

The power allocation problem is to find $p_{s}$ such that the BER in 27) is minimized subject to the power constraint by solving the following optimization problem

$$
\mathcal{L}\left(p_{s}\right)=\mathrm{BER}+\xi\left(2 p_{s}+p_{r}-p\right)
$$

where $\xi$ is a positive Lagrange multiplier. The necessary condition for the optimality is found by setting the derivatives of the Lagrangian in (29) with respect to $p_{s}$ and $p_{r}$ equal to zero, respectively. Reusing the power constraint, we can calculate at high SNR that

$$
\begin{aligned}
& p_{s}=\frac{p}{4}, \\
& p_{r}=\frac{p}{2},
\end{aligned}
$$


which indicates the power allocated in the relay should be equal to the total transmit power at both sources in order to compensate the energy used to broadcast combined information in one time slot.

\section{Simulation Results}

In this section, we provide simulation results for the proposed ANC-DM scheme. We also include corresponding coherent detection results for comparison. All simulations are performed for a BPSK modulation over the Rayleigh fading channels. The frame length is $L=100$. For simplicity, we assume that $2 p_{s}+p_{r}=p=3$, and $S_{1}, S_{2}$ and $R$ have the same noise variance $N_{0}$. The $\operatorname{SNR} \psi_{s}$ can be then calculated as $\psi_{s}=p_{s} / N_{0}$.

Fig. 2] shows the simulated BER performance for differential and coherent ANC schemes in bi-directional relaying without using constellation rotation. Equal transmit power allocation is applied: $p_{s}=p_{r}=p / 3$. It can be seen that the differential scheme suffers around 3 $\mathrm{dB}$ performance loss compared to the coherent ANC scheme, which has been validated by (19). We also include the Genie-aided result by assuming that $\mu$ is perfectly known by the source such that traditional differential decoding can be performed. It shows from the results that there is almost no performance loss using the estimation method in (14) which clearly justifies the robustness of the proposed differential decoder.

It is worthwhile mentioning that, in [6], it uses similar Genie-aided result as a benchmark as well. The major difference is that the detectors in [6] have much inferior performance than the Genie-aided result, and it has about $6 \mathrm{~dB}$ performance loss in comparison to the coherent detection scheme. However, our proposed detection algorithm has comparable performance with the genie-aided result, and only has $3 \mathrm{~dB}$ performance loss than the coherent detection results. This clearly indicates that our proposed method outperforms the differential detectors in [6]. The main performance loss in [6] is due to that uncoherent detection approach is employed by statistically averaging off the impact of channel fading coefficients ignoring the instantaneous channel state information. But, our method utilizes differential detection relying on the operation with the previous received signals which could be more adaptive to variation of the channels.

In Fig. 3, we compare the analytical and simulated BER performance of the proposed differential modulation scheme. Equal transmit power allocation is also applied by setting $p_{s}=p_{r}=p / 3$. From the figure, it can be observed that at high SNR, the analytical BER derived by (27) converged to the simulated result, which justifies the validation of (27). 
In Fig. 4, we examine the BER performance of the proposed differential modulation protocol with power allocation by setting $p_{s}=p / 4$ and $p_{r}=p / 2$ subject to the total power constraint. From Fig. 4, it can be observed that with optimal power allocation, the proposed scheme obtains about $2 \mathrm{~dB}$ performance gain in comparison with the equal power allocation scheme at high SNR. We also compare the result by another power setting: $p_{s}=0.4 p$ and $p_{r}=0.2 p$, and inferior result can be again observed compared to the optimal power allocation.

In Fig. 5, we plot the BER curves in terms of $\lambda=p_{s} / p_{r}$ defined in (20) using different noise variance $N_{0}$ with the asymptotic BER constraint in (27). With the power constraint $2 p_{s}+p_{r}=p$, the SNR can be therefore calculated by $\psi_{s}=\frac{\lambda p}{(2 \lambda+1) N_{0}}$, where $p=3$. It shows that best performance is obtained when $\lambda=0.5$. In other words, $p_{s}=p / 4$ and $p_{r}=p / 2$ is the optimal power setting between the sources and the relay, which further verify the power allocation strategy in (30). From the figure, we can also see that the asymptotic BER is very close to the simulated results for various SNR values, and they result in the same power allocation solution of $p_{s}=p / 4$ and $p_{r}=p / 2$.

In Fig 6, we examine the BER results of the proposed differential modulation scheme without and with using constellation rotation, where the signal constellation used by $S_{1}$ is rotated by $\pi / 2$ relative to that by $S_{2}$. It can be observed that the new result has very similar with the curve without rotating constellations. This indicates that using constellation rotation may not give system any gains given large frame length.

Fig. 7 shows the average normalized mean square error (MSE) of $\mu$ estimation in (14) as a function of the SNR, where the average normalized MSE is calculated as $\frac{\frac{1}{N} \sum_{k=1}^{N}(\mu(k)-\widehat{\mu}(k))^{2}}{\mathbb{E}[\mu(k)]}$ and $\widehat{\mu}(k)$ is the estimate of $\mu(k)$. It can be observed that the estimation is quite accurate, particularly at high SNR.

\section{CONCLusions}

In this paper, we have proposed a simple differential modulation scheme for bi-directional relay communications using analog network coding when neither sources nor the relay has access to channel state information. Simulation results indicate that there exist about $3 \mathrm{~dB}$ loss compared to the coherent detection scheme. Analytical BER is derived to validate the proposed method. In addition, based on the asymptotic BER at high SNR, an optimal power allocation between the sources and the relay was derived to enhance the system performance. 


\section{REFERENCES}

[1] R. Ahlswede, N. Cai, S.-Y. R. Li, and R.W. Yeung, ”Network information flow," IEEE Transactions on Information Theory, vol. 46, pp. 1204-1216, Jul. 2000.

[2] C. Yuen, W. H. Chin, Y. L. Guan, W. Chen, and T. Tee, "Bi-directional multi-antenna relay communications with wireless network coding," in IEEE Proc. Vehicular Technology Conference, pp. 1385-1388, May 2008.

[3] P. Popovski and H. Yomo, "Wireless network coding by amplify-andforward for bi-directional traffic flows," IEEE Comms Letters, vol. 11, pp. 16-18, Jan. 2007.

[4] S. Zhang, S. C. Liew, and P. P. Lam, "Hot topic: physical-layer network coding," Proc. ACM MobiCom, Los Angeles, CA, 2006.

[5] S. Katti, S. Gollakota, and D. Katabi, "Embracing wireless interference: analog network coding," Proc. ACM SIGCOMM, Kyoto, Aug. 2007.

[6] T. Cui F. Gao and C. Tellambura,"Physical layer differential network coding for two-way relay channels", Proc. IEEE GlobeCom, New Orleans, CA, 2008.

[7] J. G. Proakis, Digital communications, 4th ed. New York: McGraw-Hill, 2001.

[8] Z. Wang and G.B. Giannakis, "A simple and general parameterization quantifying performance in fading channels," IEEE Trans. Commun., vol.51, no.8, pp.1389-1398, Aug. 2003.

[9] M. O. Hasna and M. S. Alouini, "End-to-end performance of transmission systems with relays over Rayleigh-fading channels," IEEE Trans Wireless Commun., vol. 2, pp. 1126-1131, Nov. 2003.

[10] M. Abramowitz and I. A. Stegun, Handbook of mathematical functions with formulas, graphs, and mathematical tables,9th ed. NewYork: Dover, 1970. 

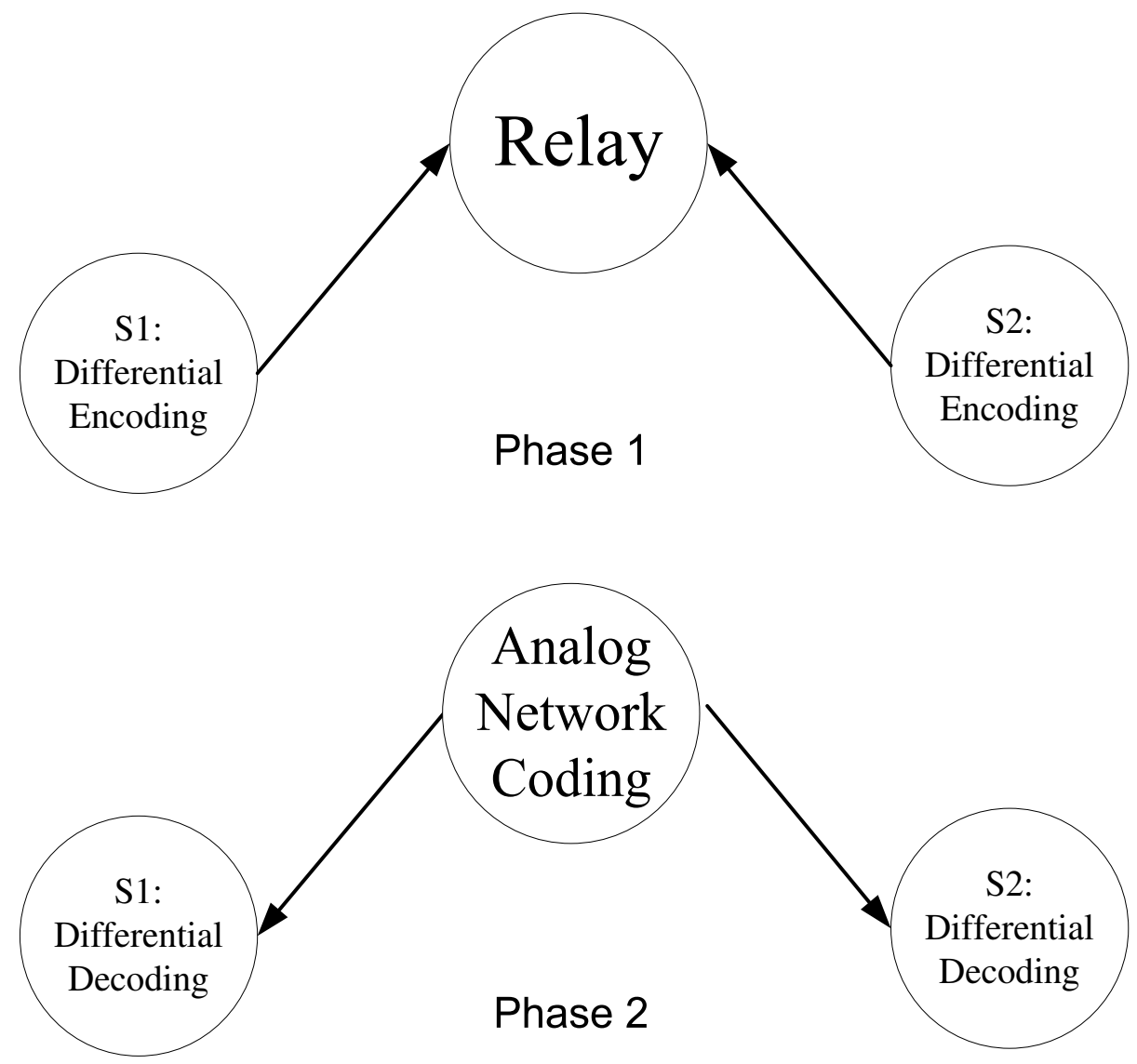

Fig. 1. Block diagram of the proposed ANC-DM scheme. 


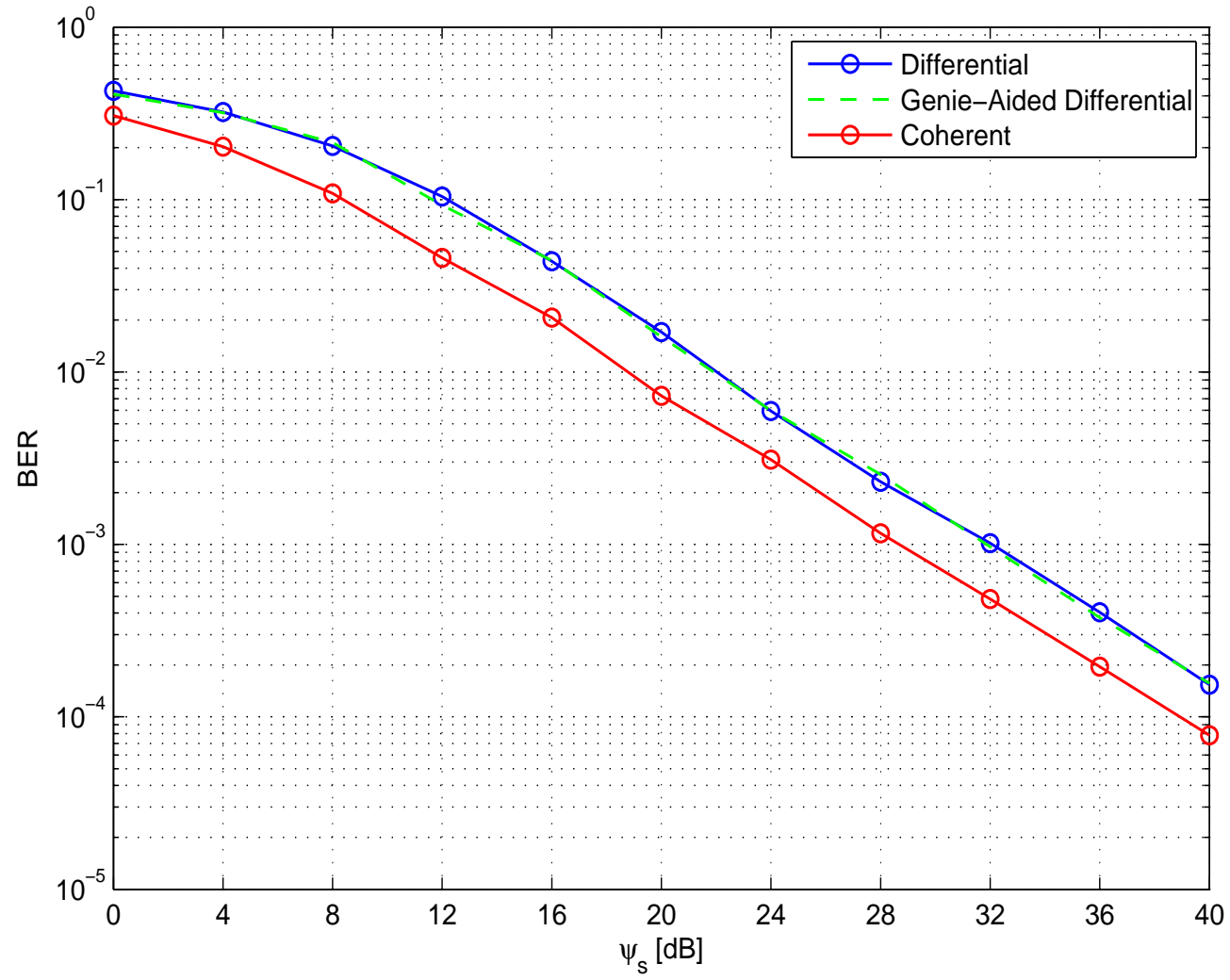

Fig. 2. Simulated BER performance by differential and coherent detections, where $p_{s}=p_{r}=p / 3$. 


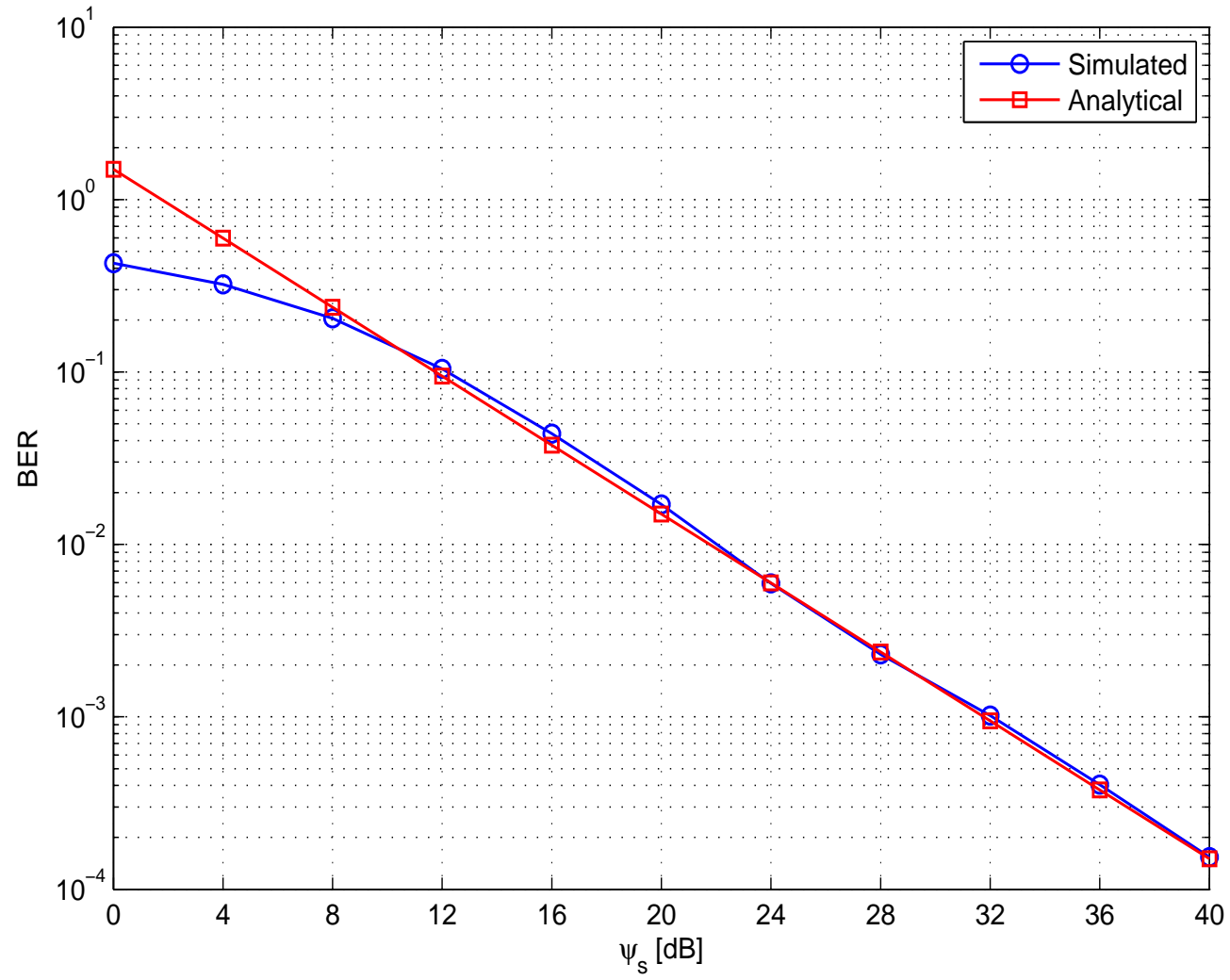

Fig. 3. Analytical and Simulated BER performance by the proposed differential scheme, where $p_{s}=p_{r}=p / 3$ 


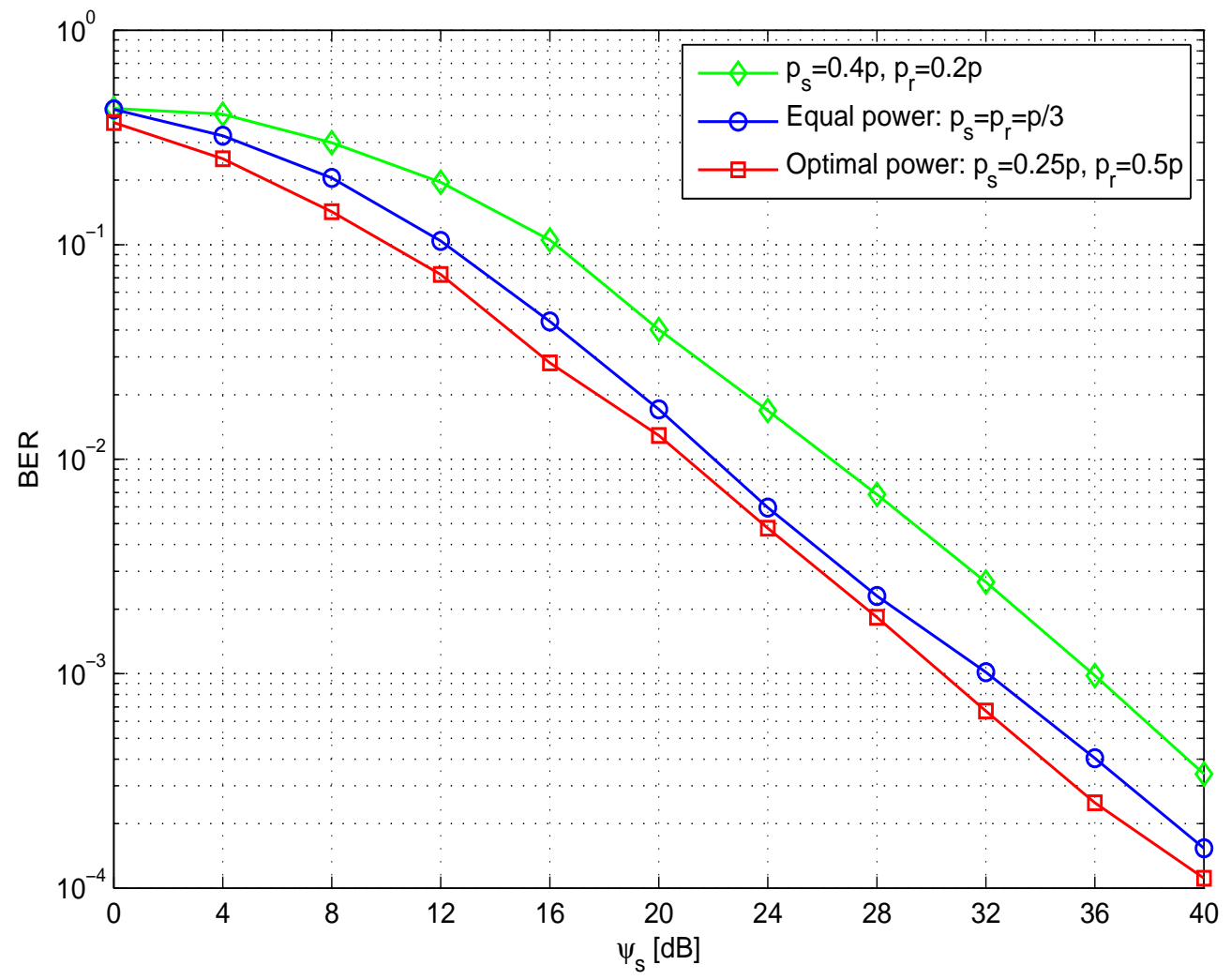

Fig. 4. Simulated BER performance by the proposed differential scheme using transmit power allocation. 


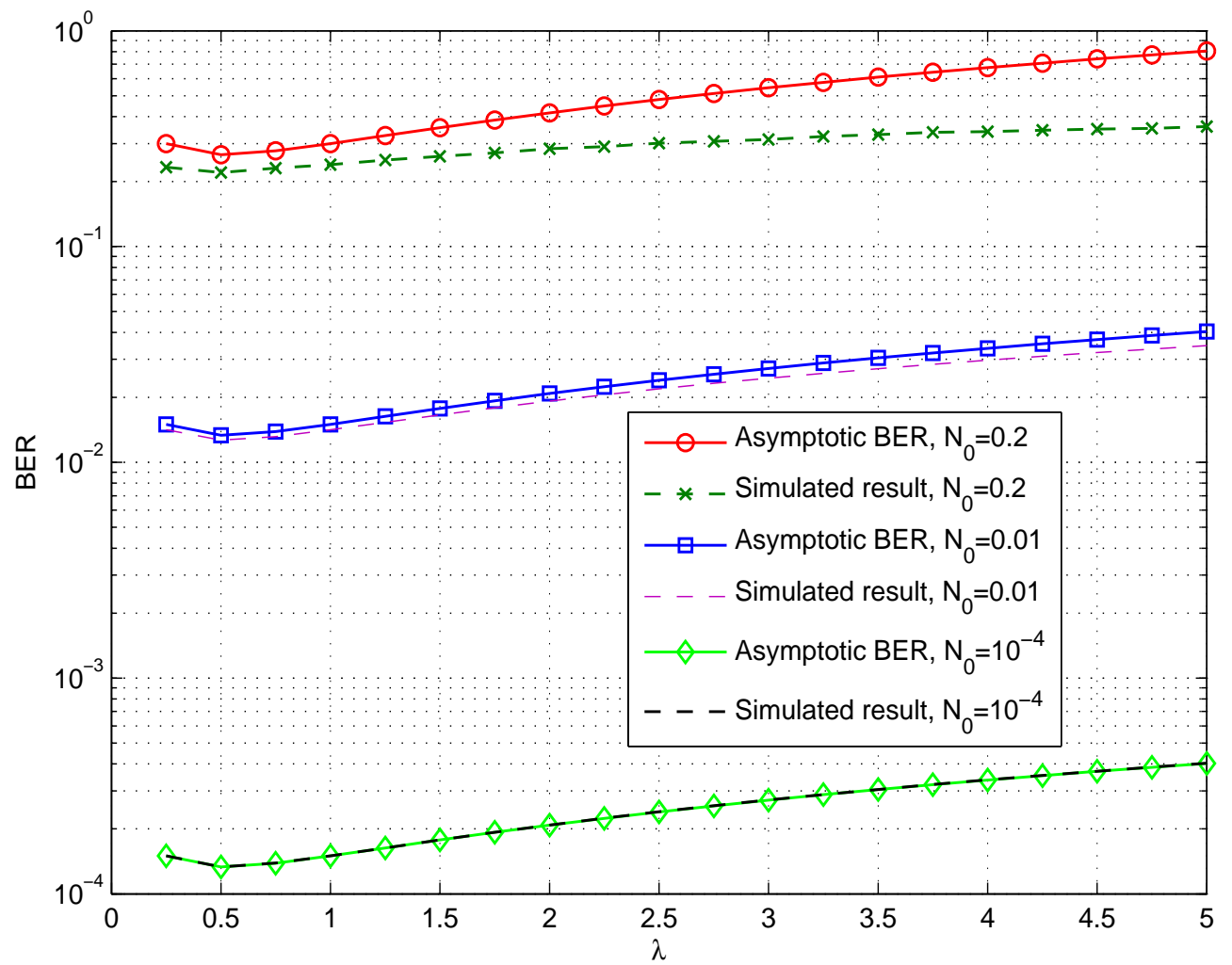

Fig. 5. Simulated BER performance by the proposed differential scheme using transmit power allocation in term of $\lambda=p_{s} / p_{r}$, with different noise variance $N_{0}$ and $\psi_{s}=\frac{\lambda p}{(2 \lambda+1) N_{0}}$. 


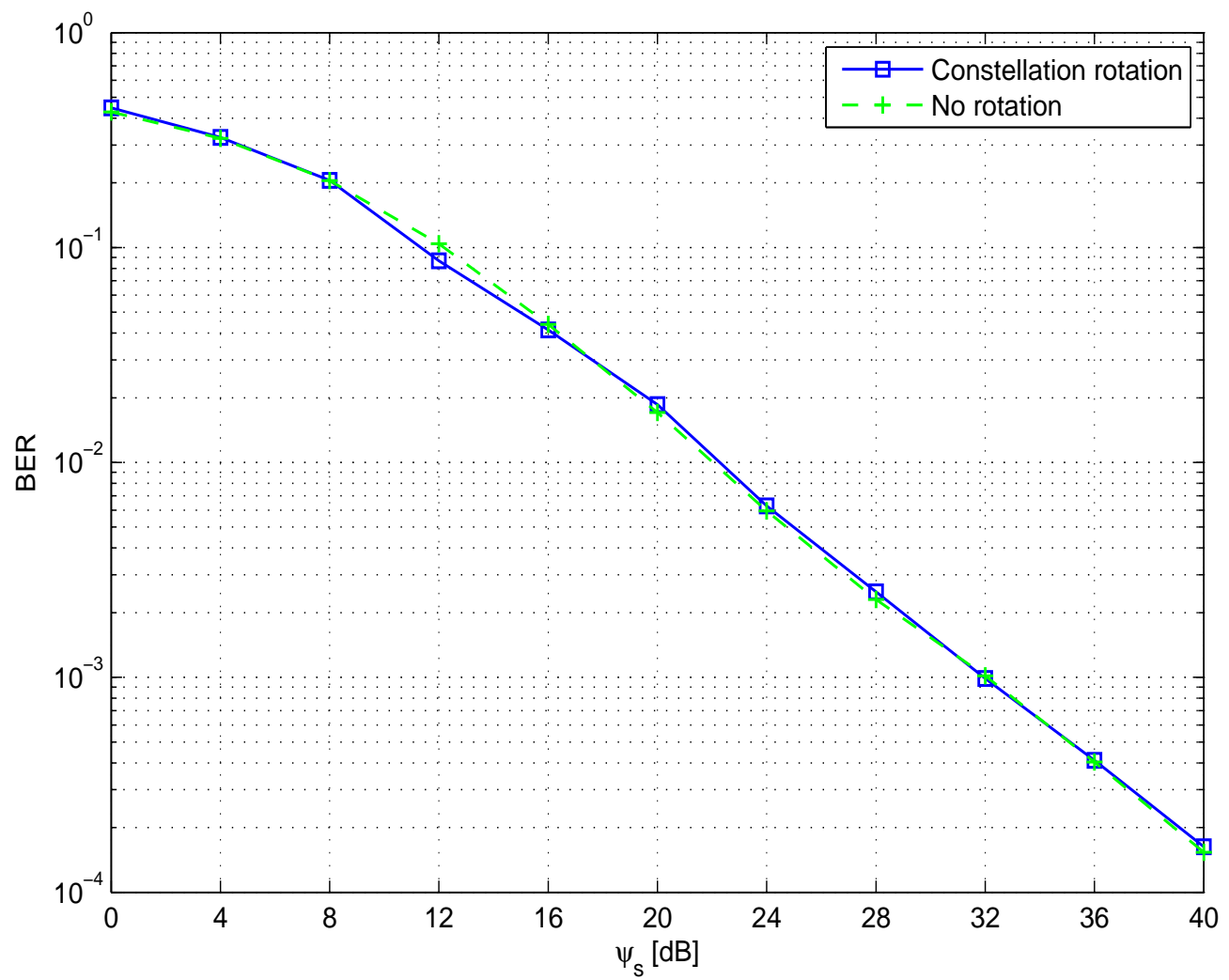

Fig. 6. Simulated BER performance by the proposed differential scheme with and without using constellation rotation, where $p_{s}=p_{r}=p / 3$. 


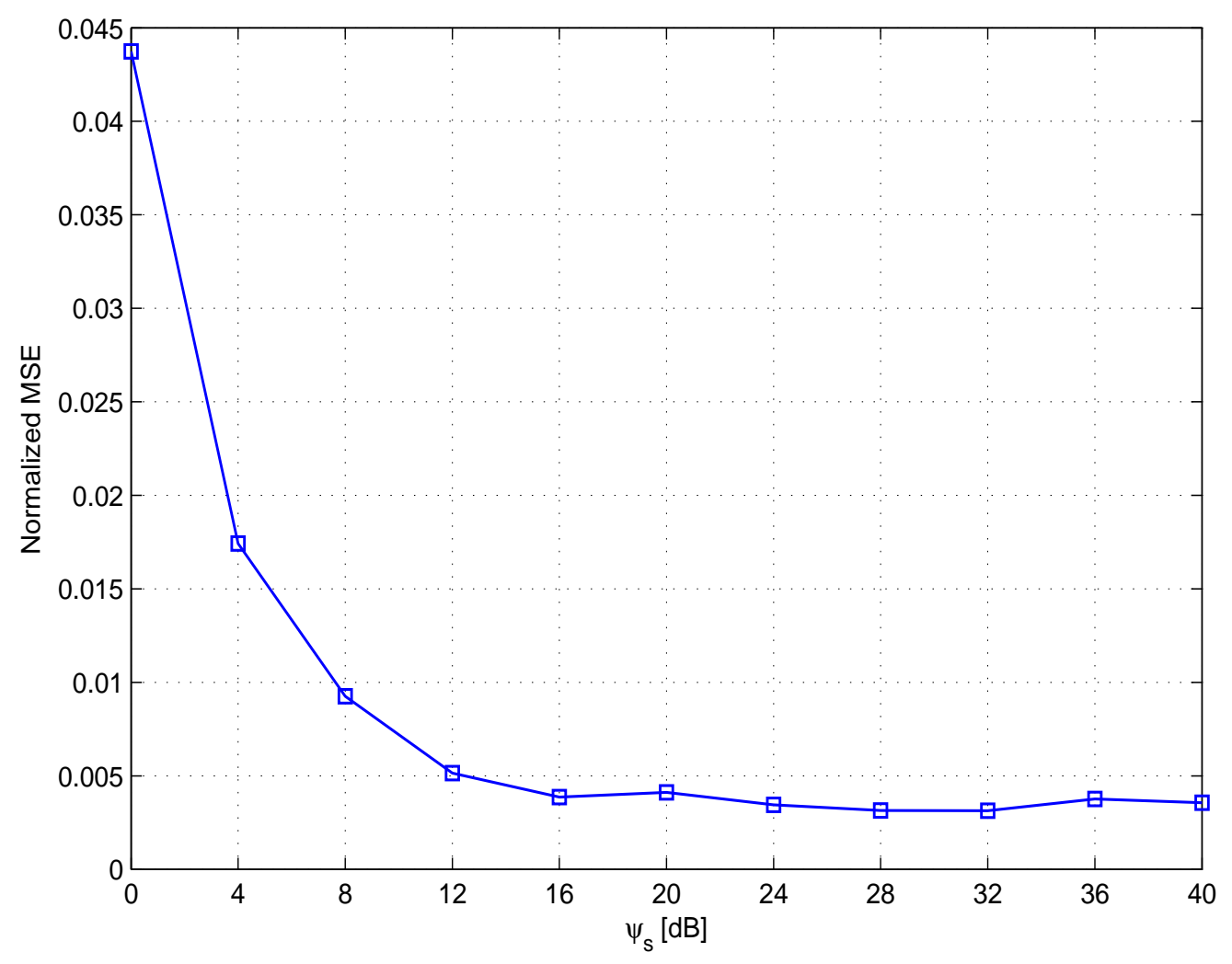

Fig. 7. The average normalized mean square error (MSE) of $\mu$ estimation in (14) as a function of the SNR. 